Review Article

\title{
Development and Application of 3D Bioprinted Scaffolds Supporting Induced Pluripotent Stem Cells
}

\author{
Dezhi Lu, ${ }^{1,2}$ Yang Liu, ${ }^{1,3}$ Wentao Li, ${ }^{1}$ Hongshi Ma, ${ }^{1}$ Tao Li, ${ }^{1}$ Xiaojun Ma, ${ }^{4}$ Yuanqing Mao, ${ }^{1}$ \\ Qianqian Liang, ${ }^{2}$ Zhenjiang $\mathrm{Ma} \oplus^{1},{ }^{1}$ and Jinwu Wang $\mathbb{1}^{1}$ \\ ${ }^{1}$ Shanghai Key Laboratory of Orthopaedic Implants, Department of Orthopaedic Surgery, Shanghai Ninth People's Hospital, \\ Shanghai Jiao Tong University School of Medicine, Shanghai 200011, China \\ ${ }^{2}$ School of Medicine, Shanghai University, Shanghai 200444, China \\ ${ }^{3}$ Spine Institute, Shanghai University of Traditional Chinese Medicine, Shanghai 200032, China \\ ${ }^{4}$ Department of Orthopedics, Shanghai General Hospital, Shanghai Jiao Tong University School of Medicine, \\ Shanghai 200080, China \\ Correspondence should be addressed to Zhenjiang Ma; mashrimp@163.com and Jinwu Wang; jinwu_wang@163.com
}

Received 7 August 2020; Accepted 6 August 2021; Published 13 September 2021

Academic Editor: Bruna Sinjari

Copyright (C) 2021 Dezhi Lu et al. This is an open access article distributed under the Creative Commons Attribution License, which permits unrestricted use, distribution, and reproduction in any medium, provided the original work is properly cited.

Three-dimensional (3D) bioprinting is a revolutionary technology that replicates 3D functional living tissue scaffolds in vitro by controlling the layer-by-layer deposition of biomaterials and enables highly precise positioning of cells. With the development of this technology, more advanced research on the mechanisms of tissue morphogenesis, clinical drug screening, and organ regeneration may be pursued. Because of their self-renewal characteristics and multidirectional differentiation potential, induced pluripotent stem cells (iPSCs) have outstanding advantages in stem cell research and applications. In this review, we discuss the advantages of different bioinks containing human iPSCs that are fabricated by using 3D bioprinting. In particular, we focus on the ability of these bioinks to support iPSCs and promote their proliferation and differentiation. In addition, we summarize the applications of $3 \mathrm{D}$ bioprinting with iPSC-containing bioinks and put forward new views on the current research status.

\section{Introduction}

The lack of tools for assessing promising drug targets impedes the development of treatments for various conditions, such as spinal cord injury and cardiovascular diseases. In addition, in some critical circumstances, such as organ transplantation, it is difficult to overcome the limited supply of suitable organ donors and a possible immune response to the organ transplant [1]. Therefore, approaches that enable tissue engineering for the development of innovative and effective treatments have been receiving increasing attention.

Three-dimensional (3D) bioprinting systems are promising tools for generating functional organs or tissues, which can be used for studying tissue morphogenesis, therapeutic drug screening, and possible organ regeneration in the future [2]. Bioprinting permits highly precise and accurate fabrication of biological 3D constructs containing cells, extracellular matrix scaffolds, and biochemical factors. Such $3 \mathrm{D}$ constructs can better mimic the human in vivo microenvironment than $2 \mathrm{D}$ cell culture environments and animal models [3].

Nevertheless, it has been reported that some cells may be damaged during the printing protocol due to shear stress [4]. Therefore, suitable bioinks and bioprinters need to be chosen to support the cells. Popular bioinks include some naturally derived biomaterials, such as polysaccharides, fibrin, and collagen, which have excellent biocompatibility. In addition to the natural scaffolds, some synthetic components can be also designed with tunable mechanical and degradation properties.

Various adult cells, such as mesenchymal stem cells, chondrocytes, cardiomyocytes (CMs), and endothelial cells, 
can be used for bioprinting. However, there are many limitations in the application of these adult cells in the repair of tissues and organs or the construction of in vitro models. First, bioprinting requires a large number of cells, and adult cells have a limited ability to proliferate. Secondly, autologous cells are difficult to obtain, whereas allogeneic cells may be rejected by the immune system.

Human-induced pluripotent stem cells (hiPSCs) have attracted global attention since their development a decade ago [5]. Because of their self-renewal properties and potential for multilineage differentiation, iPSCs may be used to generate a large number of autologous adult cells. Furthermore, the use of iPSCs allows to avoid ethical problems associated, for example, with the use of embryonic stem cells (ESCs) in stem cell research and various biomedical applications. In addition, using iPSCs to reconstruct tissues and organs in vitro better simulates normal and pathological processes, and thus, these cells serve as better research models. A noticeable challenge pertaining to the use of iPSCs in bioprinting is the multivariate nature of the differentiation process. Therefore, apart from minimizing the exposure to stress and harmful forces during the printing process, it is also important to provide the appropriate culture conditions to fabricate the desired differentiated cellular products [3]. There has been a significant interest in mimicking the 3D cytoarchitecture of native tissues in vitro and developing bioactive, biocompatible, and mechanically tunable $3 \mathrm{D}$ configured bioinks that can be used for stem cell research and therapy [6].

Considering that several reviews devoted to $3 \mathrm{D}$ bioprinting of hiPSCs have been published recently, in the present review, we focus on different bioinks prepared by $3 \mathrm{D}$ bioprinting and discuss their advantages in supporting and promoting hiPSC proliferation and differentiation. In addition, we discuss the complex $3 \mathrm{D}$ printed microstructures developed to more closely mimic human physiology. We also review the use of hiPSCs in combination with $3 \mathrm{D}$ bioprinting for the treatment of neurological, orthopedic, cardiovascular, and hepatic disorders. Finally, in our concluding remarks, we summarize these recent advances and provide an outlook for the future development of these methods.

\section{Popular Bioinks Supporting hiPSC Growth}

HiPSCs can be seeded onto or cast within various supporting biomaterials. In addition, via 3D bioprinting, constructs containing hiPSCs can be directly fabricated by using a single-step approach to generate 3D cellularized scaffolds. Ho et al. describe neural induction of PU (a thermoresponsive synthetic hydrogel) encapsulated human hiPSCs, neither reported neurite outgrowth nor cell functionality [7, 8]. In the latter case, iPSCs can be closely integrated with biomaterials by encapsulation for direct and complete contact with the extracellular elements that mimic the native cell microenvironment. Some of the reported novel bioinks are presented below (Table 1).

However, compared with adult cells, such as chondrocytes, bone marrow mesenchymal stem cells, and endothelial cells, iPSCs, especially undifferentiated ones, are more sensi- tive. Depending on the bioprinting method used, the cells are exposed to high shear forces, radiation-induced damage, and electric or thermal stress during the printing process. All these factors have a marked impact on the proliferation and differentiation potential of iPSCs; thus, it is critical to choose the appropriate bioinks and bioprinting technology.

Koch et al. conducted several studies to investigate the effect of biomaterials on cell survival, pluripotency, and differentiation [12]. The laser bioprinting process itself had only a minor effect on cell survival. Survival rates of hiPSCs were higher if they were grown in Matrigel, blood plasma, and hyaluronic acid than in alginate, fibrin, collagen, or Geltrex. Notable cell apoptosis within $10 \mathrm{~h}$ may be ascribed to dissociation-induced cell death. After $24 \mathrm{~h}$, high cell survival rates were observed when bioink medium or fibrinogen solution was used, each mixed with hyaluronic acid to achieve appropriate viscosity for bioprinting and avoid rinsing the cells. Importantly, the properties of hiPSCs within sols or gels were different from the characteristics of hiPSCs on the surface of bioinks.

Polysaccharide-based bioinks that contain polysaccharides, such as alginate, agarose, and chitosan, are widely used because they exhibit favorable biocompatibility, require mild cross-linking conditions, and have limited impact on iPSC properties. A polysaccharide-based bioink consisting of agarose, alginate, and carboxymethyl-chitosan was designed to support hiPSC expansion and differentiation in the clinical setting. Then, this bioink was cross-linked in calcium chloride to obtain a stable and porous construct. Such printed construction favored homogenous distribution and high viability of hiPSCs. After gelation, iPSCs could be maintained as self-renewing stem cells within the printed bioinks, and cell proliferation persisted for more than 9 days [14]. Shu et al. fabricated a 3D printed alginate tube structure (approximately $13 \mathrm{~mm}$ tall) composed of sodium alginate $(1.5 \% \mathrm{w} / \mathrm{v})$ and containing $600 \mathrm{mM}(6 \%)$ of calcium chloride solution prepared in Millipore water. They showed that hiPSCs could be bioprinted with their valve-based printing process without adverse effects on their viability and pluripotency [15]. However, many of these polysaccharides are markedly fragile, lacking sufficient mechanical strength to be retained in the transplant tissue site, and often experiencing low mechanical properties.

2.1. Fibrin-Based Bioinks. Fibrin is a hydrogel formed by the enzymatic reaction between thrombin and fibrinogen-the key proteins involved in blood clotting-which supports extensive cell growth and proliferation [16]. Recently, fibrin-based bioinks have also been used as naturally derived biomaterials for supporting cultured stem cells and their differentiation into specific tissues [17, 18]. Sharma et al. designed a novel fibrin-based bioinks combined with drugreleasing guggulsterone microspheres and hiPSC-derived neural progenitor cells. The construct was printed as domes with a $1 \mathrm{~cm}$ diameter using a microfluidics-based RX1 bioprinter. Guggulsterone microspheres were used for controlled drug release; they also facilitated the survival of hiPSCs in bioprinted tissue and supported hiPSC differentiation into dopaminergic neurons [10]. The rapid 
TABLE 1: Novel 3D printed bioinks and their applications in tissue engineering.

\begin{tabular}{|c|c|c|c|}
\hline Bioinks & Applications & Bioprinting methods & References \\
\hline $\begin{array}{l}\text { Gelatin methacrylate }(5 \% \mathrm{wt} / \mathrm{vol})+\text { glycidyl } \\
\text { methacrylate-hyaluronic acid + hiPSCs }\end{array}$ & Liver tissue & $\begin{array}{l}\text { Digital light processing-based } \\
\text { 3D printing }\end{array}$ & Ma et al. [9] \\
\hline $\begin{array}{l}\text { Fibrin }(20 \mathrm{mg} / \mathrm{mL}) \text {, alginate }(5 \mathrm{mg} / \mathrm{mL}) \text {, } \\
\text { and genipin }(0.3 \mathrm{mg} / \mathrm{mL})+\text { hiPSCs }\end{array}$ & Neural tissue & $\begin{array}{l}\text { Microfluidics-based } \\
\text { RX1 bioprinter }\end{array}$ & Abelseth et al. [10] \\
\hline $\begin{array}{l}\text { Hydroxypropyl chitin + bioactive } \\
\text { Matrigel + hiPSCs }\end{array}$ & $\begin{array}{l}\text { 3D microtissue differentiation } \\
\text { and drug screening }\end{array}$ & $\begin{array}{l}\text { Extrusion-based } \\
\text { 3D bioprinting }\end{array}$ & Li et al. [11] \\
\hline $\begin{array}{l}\text { Agarose }+ \text { alginate + carboxymethyl } \\
\text { chitosan }+ \text { iPSCs }\end{array}$ & Neural tissue & 3D bioprinting & Gu et al. [12] \\
\hline $\begin{array}{l}\text { hiPSC-derived CMs + human umbilical vein } \\
\text { endothelial cells + human adult ventricular } \\
\text { cardiac fibroblasts to form mixed cell spheroids }\end{array}$ & Cardiac patches & 3D bioprinter & Ong et al. [13] \\
\hline
\end{tabular}

degradation rate of pure fibrin is a major limitation for its use in differentiating hiPSCs for tissue engineering applications. Therefore, Robinson et al. designed a fibrin-based bioinks containing genipin, a natural cross-linking agent. They showed that genipin improved neuronal differentiation of neural progenitors derived from hiPSCs in 2D culture, having a concentration-dependent effect on the morphology and mechanical performance of 3D fibrin scaffolds [19]. The optimal concentration of genipin for its maximal neurotrophic and fibrin cross-linking effects ranged between 1 and $2.5 \mathrm{mM}$. Ruchi et al. show how our novel fibrin-based bioink formulation combined with drug releasing microspheres can serve as a tool for bioprinting tissues using hiPSC. They demonstrate that use a microsphere-laden bioink to bioprint hiPSC can promote the differentiation of neural tissue [20].

2.2. Hyaluronic Acid-Based Bioinks. Differentiation of stem cells into specific lineages during development is tightly regulated by the local microenvironment that includes growth factors, extracellular matrix proteins, and surrounding cells. Hyaluronic acid is one of the main components of the extracellular matrix, and hybrid scaffolds composed of hyaluronic acid and collagen can be used for cartilage regeneration. As the collagen concentration increases, the tensile strength performance of the scaffolds improves, and their degradation period increases [21]. Kupfer et al. optimized a photocrosslinkable formulation of native extracellular matrix proteins to promote the differentiation of iPSCs into CMs and used this bioink for the 3D printing of hiPSC-laden structures with two chambers and a vessel inlet and outlet [22].

2.3. Bioinks Based on Synthetic Components. In addition to the naturally derived biomaterials, synthetic components have been studied with respect to their utility as components of $3 \mathrm{D}$ printed structures. $\mathrm{Li}$ et al. investigated the feasibility of printing hiPSCs in a primed state after dissociation into single cells by using an extrusion-based 3D bioprinting method. The new bioink material, hydroxypropyl chitin, was introduced and mixed with bioactive Matrigel to form bioinks with different composition ratios [11]. Kerscher et al. designed a synthetic bioinks, consisting of polyethylene glycol and fibrinogen, which could be used to encapsulate
hiPSCs, guide their differentiation, and promote the process of functional cardiac tissue formation [23]. Compared with bioinks composed of natural biomaterials or purely synthetic materials, such hybrid composite bioinks may have both good biocompatibility and tunable mechanical properties. Kerscher et al. designed two alginate/gellan gum/laminin (ALG/GG/LAM) hydrogel blends that are presented for the fabrication of hiPSC-based 3D neural models. Due to their wide range of applications, adjustability, and printing capabilities, the ALG/GG/LAM-based 3D neural models are of great potential for $3 \mathrm{D}$ neural modeling in the future [24].

\section{Effect of the Micro/Nano Structure on hiPSC- Derived 3D Printed Tissue Model}

Recently, novel methods have been used to fabricate complex 3D printed microstructures that could mimic human physiology more closely. Sophisticated 3D scaffold arrangements or micropatterning by 3D bioprinting favor tissue meddles to achieve high predictability and low cost efficiency [25].

$\mathrm{Ma}$ et al. presented a patterned biomimetic hiPSCderived hepatic model with microscale hexagonal units of liver cells and supporting cells created by using a 3D bioprinting method (Figure 1) [9]. First, 5\% (wt/vol) gelatin methacrylate and $2 \%$ (wt/vol) glycidyl methacrylatehyaluronic acid at a $1: 1$ ratio were chosen to support the cells. Then, the micropatterns, which mimicked the hepatic lobule structure, were transferred to hydrogels using the digital light processing-based 3D bioprinting method. To photopolymerize the hydrogel solutions, a digital micromirror device chip was used to generate photo-masks according to the input digital patterns. The entire process was completed in several seconds under minimal UV illumination. Compared with the features of the $2 \mathrm{D}$ monolayer culture, this $3 \mathrm{D}$ biomimetic liver model had improved morphological organization and exhibited increased secretion of the metabolic product, upregulated expression of the liverspecific genes, and enhanced cytochrome P450 induction in hiPSC-derived hepatic progenitor cells (HPCs).

Ong et al. created biomaterial-free cardiac tissue using hiPSC-derived CMs by using a 3D bioprinting method [13] (Figure 2). In a sterile environment, the cardiospheres were picked up, transferred, and loaded onto a needle array 


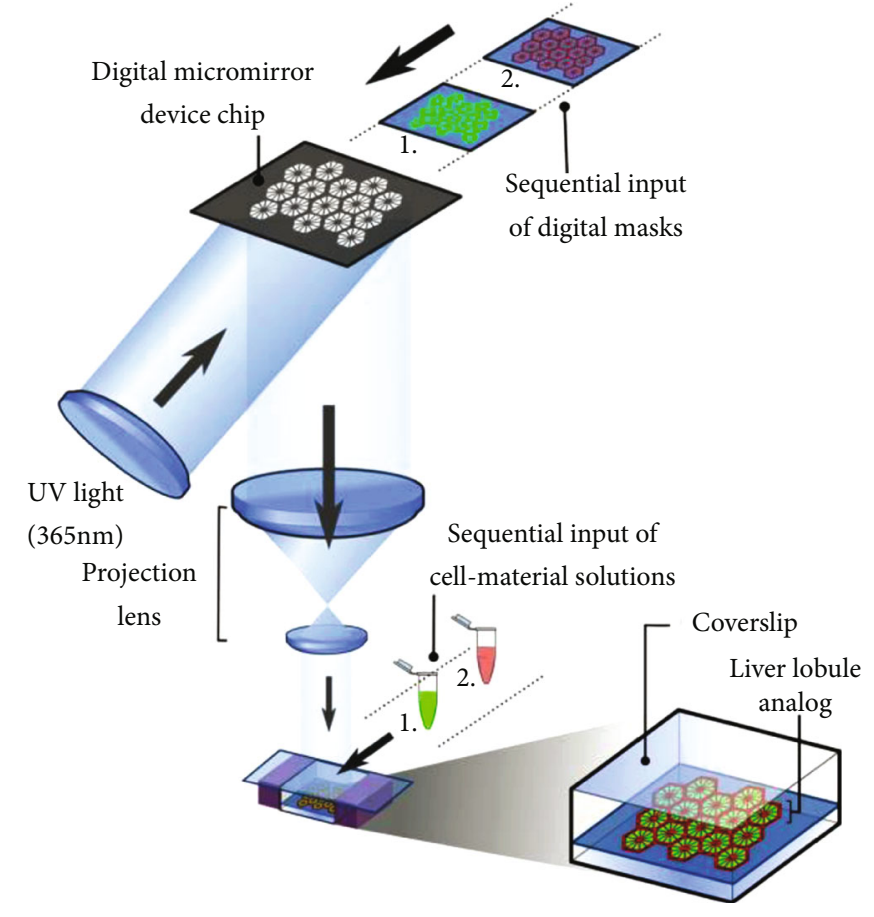

(a)
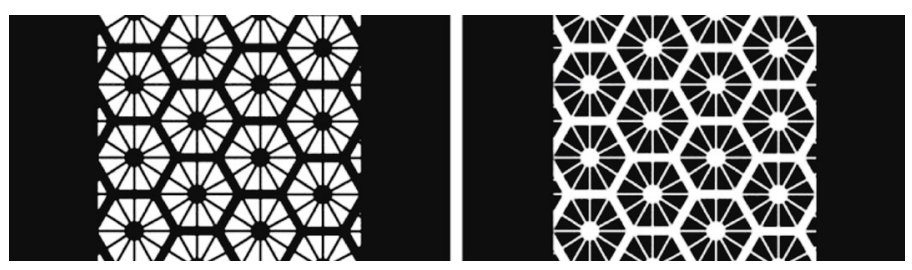

(b)

Figure 1: Continued. 


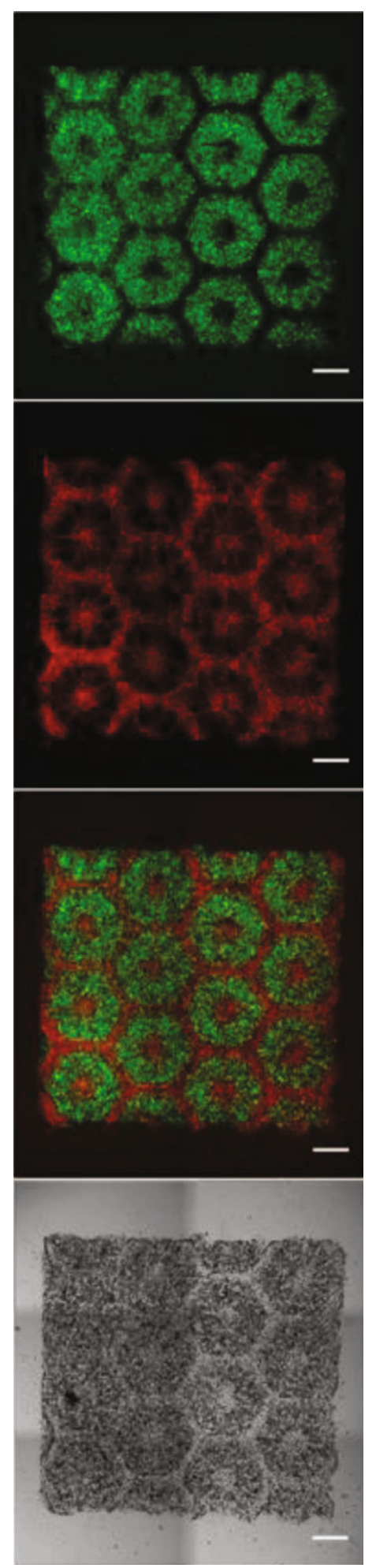

(c)

FIGURE 1: 3D bioprinting of a hydrogel-based hepatic construct with complex 3D printed microstructure that more closely mimics the physiological properties of the human liver [9].

individually by a robotic arm, in exact spatial coordinates with the help of specific 3D design software. Using this method, cardiospheres could be positioned in precise coordinates and flexible grid configurations, with uniform shape and thickness, which enabled to obtain a more uniform tissue structure at both the macroscopic and microscopic levels than afforded by random self-assembly of cardiospheres [26]. Park et al. also developed 3D cardiac tissue derived 


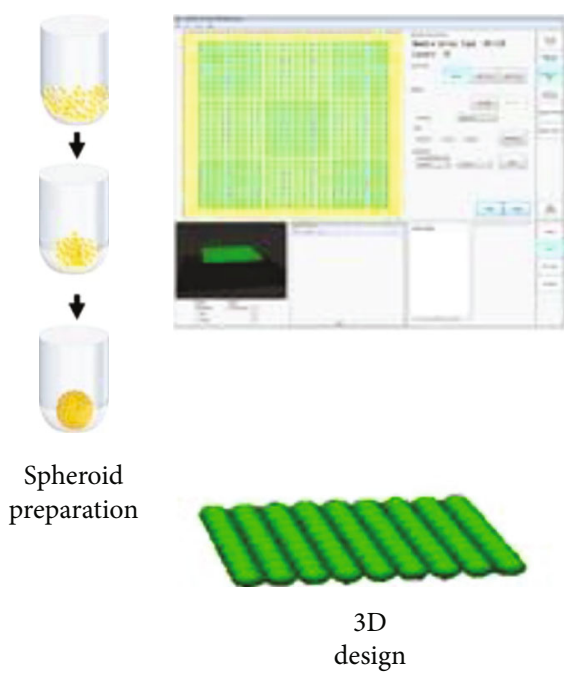

(a)

(b)

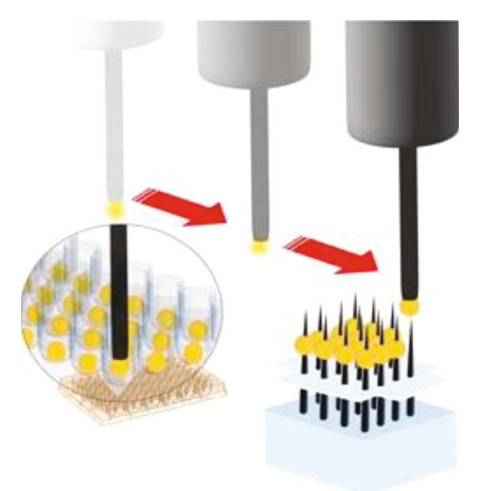

3D Bioprinting on a needle array

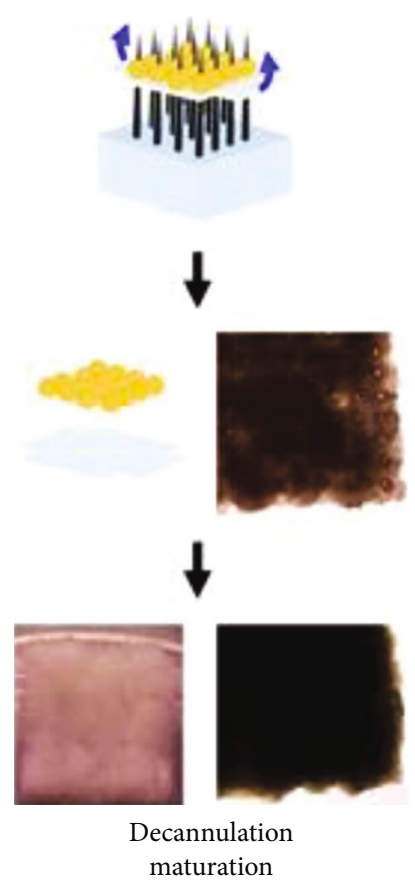

(d)

FIGURE 2: Schematic overview of biomaterial-free cardiac 3D bioprinting process [13].

from hiPSCs by using 3D bioprinting on a needle array (Figure 3). Importantly, they built a computational model to visualize the $3 \mathrm{D}$ stress distribution to investigate the mechanical reliability of implantable tissues. Their method could serve as a nondisruptive framework that measures the contractility, beating patterns, and viscoelasticity of implantable cardiac tissues to be used in heart tissue regeneration [1].

To solve the problem of producing structures of the size at which individual cells interact, the multiphoton-excited $3 \mathrm{D}$ printing method was used to fabricate native-like extracellular matrix bioscaffolds with submicron resolution [27]. The scaffolds were seeded with smooth muscle cells, endothelial cells, and CMs (at a 1:1:2 ratio), which had been differentiated from hiPSCs to generate hiPSC-derived cardiac muscle patches.

\section{3D Bioprinting of iPSCs in Tissue Engineering}

In recent years, multiple sources of iPSCs and biomaterials have been used in combination with bioprinting techniques in tissue engineering. The field of iPSC bioprinting is evolving rapidly.

4.1. Applications for Nerve Tissue Engineering. Millions of people worldwide are affected by nerve injuries that cause incapacitation due to permanent cognitive impairment, movement disabilities, and psychiatric problems, leading to increased economic burdens for the society and reduced quality of life $[28,29]$. The emerging tissue-engineered nerve grafts can replace traditional nerve anastomosis as a treatment for nerve injury [30]. Several studies have attempted to understand the molecular pathogenesis of neurological disorders. hiPSC bioprinting technologies bring a change in the strategy of treating the structural damage to the nervous system. During the past decade, stem cells have been shown to have a potential for the repair of the impaired nervous system [31]. The iPSCs can be differentiated into neural crest or neural progenitor cells, which can subsequently be patterned to different neuron subtypes including glutamatergic, GABAergic, cholinergic, and dopaminergic neurons [32-34]. Stem cells secrete large amounts of cytokines and growth factors to induce the activity of local tissue progenitors and stromal cells, thereby promoting tissue repair [35].

$3 \mathrm{D}$ printing of the iPSC-laden bioinks generated scaffolds containing consistently distributed stem cells. Bioinks including iPSCs, alginate, carboxymethyl chitosan, and agarose were squeezed and printed by Gu et al. [14] (Figure 4). The cells encapsulated in bioinks were continuously viable, the proportion of dead cells was almost negligible, and their activity remained high by prolonging in excess of 7 days. During the process of maintaining constructs for stem cell expansion, hiPSCs formed aggregates that generated large spheroids on day 7. This spherical state was similar to colony formation in the conventional 2D culture. Interestingly, the spheres in the $3 \mathrm{D}$ culture system contained tightly packed cell clusters, which were markedly different from the sharp, flat edges, and tight accumulation during the conventional $2 \mathrm{D}$ culture. In addition, immunophenotype identification by confocal microscopy showed that iPSC spheroids within the constructs expressed OCT4, SOX2, SSEA4, and TRA-160. Consequently, iPSCs in situ differentiated into embryoid bodies containing endoderm, ectoderm, and mesoderm cells. With the addition of neural induction/differentiation 


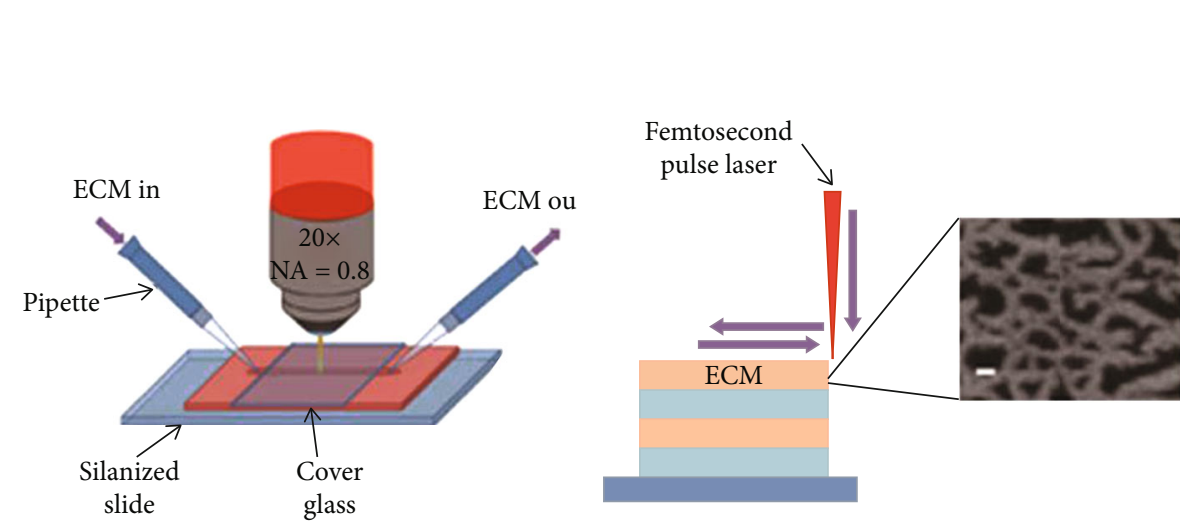

(a)

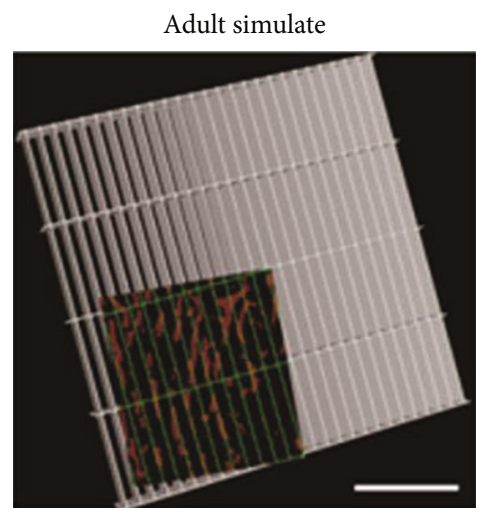

(c)

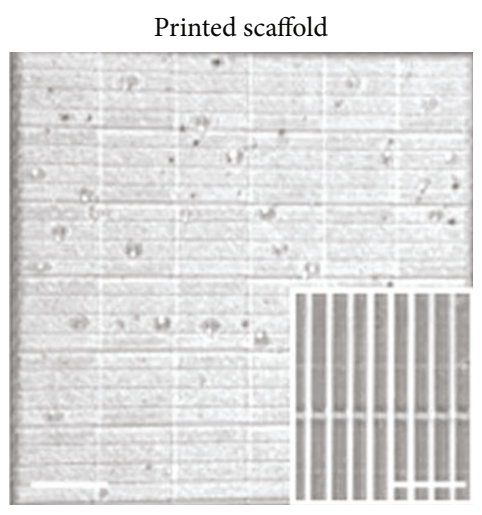

(d)

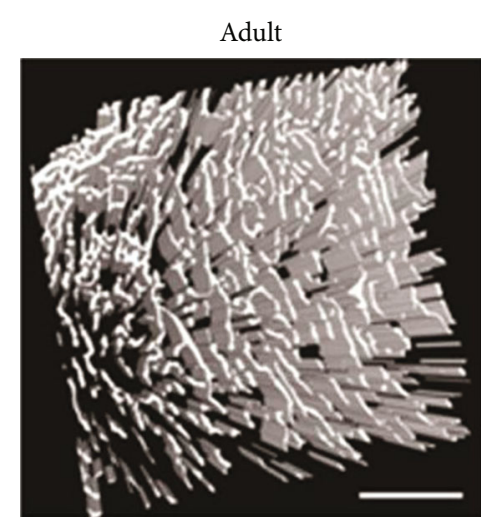

(b)

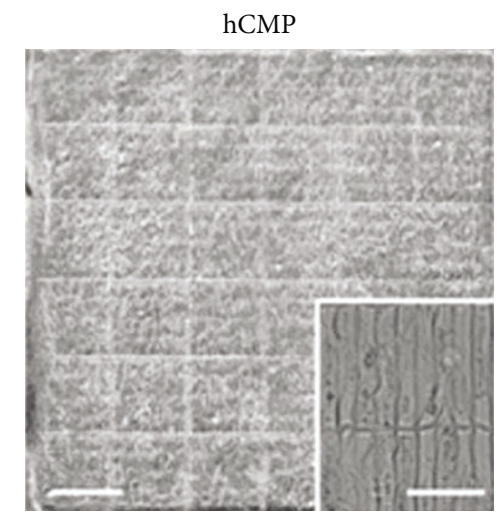

(e)

FIGURE 3: Human-induced pluripotent stem cell-derived cardiac muscle patch (hCMP) fabrication by 3D multiphoton excited printing [27]. ECM: extracellular matrix.

mediators, iPSC constructs can produce more homogeneous nerve tissue with neurons and supporting neuroglia. Minoru et al. develop an hiPSC-sensory neuron (SN) laden bioinks using highly purified and functional $\mathrm{SN}$ populations to $3 \mathrm{D}$ bioprint microarchitecture wirings that demonstrate responsiveness to warm/cold sense-inducing chemicals and mechanical stress. By a bioprinting technique, the hiPSCSNs were seeded into the hollow microchannels created by sacrificial gelatin ink printed in the GelMA supporting bath; this biofabrication approach could be amenable to incorporate sensible SN networks into the engineered skin equivalents that have the potential to regenerate sensible functions by connecting host neuron systems in injured areas [36].

4.2. Applications for Bone Tissue Engineering. Osteoarthritis was once considered a degenerative disease of the joint. In recent years, it has been shown that this disease is caused by mechanical trauma, inflammation, and other factors that cause cartilage lesions. Cartilage components eliciting an autoimmune response cause secondary articular cartilage destruction [37, 38]. The mechanism is summarized as follows: (1) inhibition of proteoglycan synthesis and destruction of collagen fibers induce loss of articular cartilage elasticity, (2) increasing hydraulic permeability leads to increase compressive stress on chondrocytes, and (3) decomposing enzymes further destroy and reform the surface of articular cartilage [39, 40].

At present, autologous chondrocyte implantation and autologous cartilage tissue transplantation techniques are widely used in clinical practice. Although chondrocyte implantation can regenerate hyaline cartilage-like tissues, the mechanical function and durability of the newly generated articular surface are still suboptimal. Owing to the difference in thickness, texture, structure, and biomechanical properties of the cartilage, there are still problems with respect to selecting the chondrocyte donor area to adapt to the repair of cartilage defects in different parts of the joint $[41,42]$. In addition, patients often need to perform surgical operations twice, and healing frequently depends on the quality and quantity of the autologous cells of the patient.

Tissue engineering of articular cartilage has made considerable progress. Bioprinted cartilage substitutes are used for the treatment of secondary knee osteoarthritis, articular joint injuries, and articular cartilage degeneration, which indicate their high clinical translation potential and demand $[43,44]$. Mesenchymal stem cells can differentiate into cells of the mesodermal lineage, giving rise to numerous specialized connective tissues, including the bone, adipose tissue, and cartilage. In contrast to transplanted chondrocytes, mesenchymal stem cells preferentially differentiate into the bone. Concurrently, the composition of the bioinks in the 


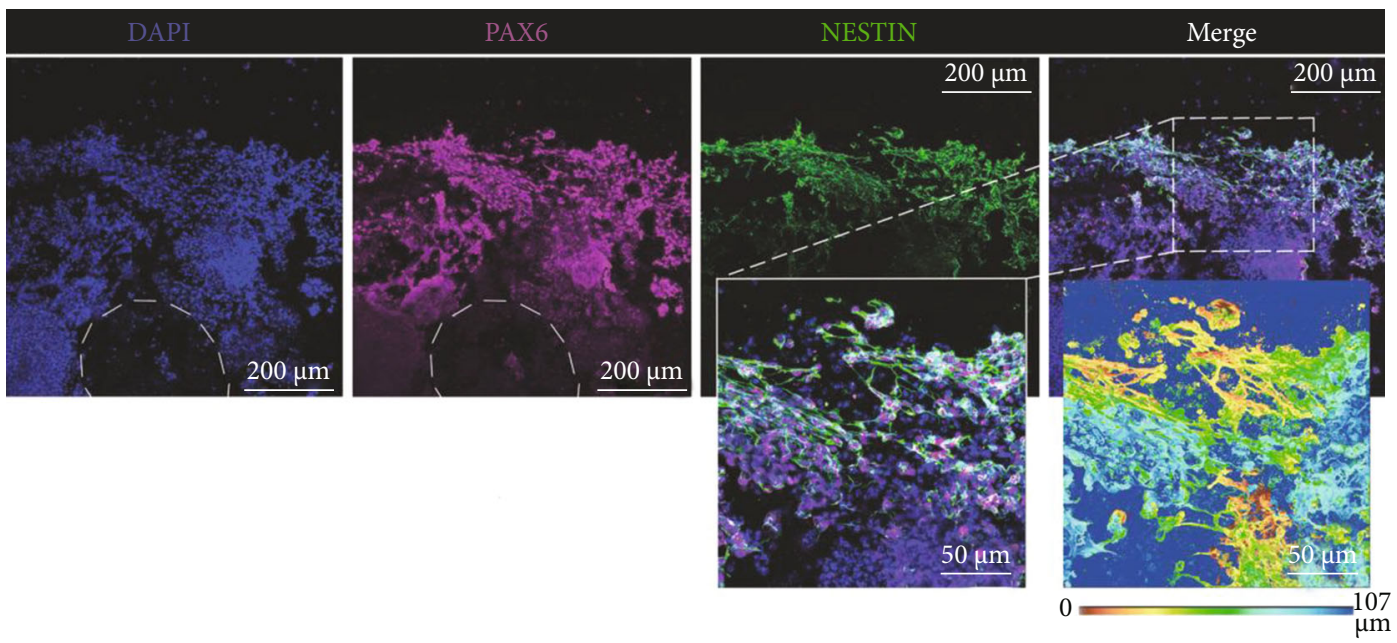

FIGURE 4: Immunophenotyping of 3D bioprinted hiPSCs 20 days after printing, including 17 days of neural induction [12].

process of biomanufacturing is not only important to ensure the long-term activity of hiPSCs and maintenance of the 3D structure but also to provide an appropriate physiological simulation environment after differentiation. Two bioinks have been recently investigated: nanofibrillated cellulose (NFC) with alginate (NFC/A) and NFC with hyaluronic acid (NFC/HA). The use of the NFC/A bioinks was associated with enhanced levels of cellular activity [45]. To induce directional cartilage differentiation, hiPSCs and irradiated mature chondrocytes were bioprinted in NFC/A and NFC/HA bioinks. After 5 weeks, hyaline-like cartilaginous tissue with collagen type II expression and lacking tumorigenic OCT4 expression was observed in 3D bioprinted NFC/A constructs. Moreover, a marked increase in cell number within the cartilaginous tissue was detected by 2-photon fluorescence microscopy, indicating the importance of high cell densities for achieving favorable survival after printing. It was concluded that NFC/A bioink was suitable for bioprinting iPSCs to support cartilage production in cocultures with irradiated chondrocytes. However, the time and concentration of chondrogenic factors need to be further optimized. In the future, the use of iPSCs for 3D bioprinting will be a potential treatment for repairing cartilage after joint damage. However, more studies are necessary to further optimize and improve the bioprinting scheme and obtain the functional proof of the newborn cartilage.

4.3. Application for Cardiovascular Tissue Engineering. Myocardial infarction seriously endangers human health. In the field of cardiovascular disease treatment, the fact that myocardial tissue lacks self-repairing ability is a serious challenge [46]. Traditional treatments, such as pharmacological therapy, angioplasty, and coronary artery bypass surgery, cannot restore necrotic and fibrotic damaged myocardium to the normal state $[47,48]$. In recent years, it has been found that stem cell transplantation can repair diseased myocardium [49]. Since the very first engineered heart tissues were introduced more than two decades ago, a wide array of hiPSCderived cardiac spheroids, organoids, and heart-on-a-chip models has been developed incorporating the latest available technologies and materials [50].

hiPSCs have emerged as a key component of cardiac tissue engineering. Scaffold-free cellular spheroids obtained from a coculture of hiPSC-derived CMs, fibroblasts, and endothelial cells were 3D printed, and these cardiac cellular patches were tested successfully in rat models of myocardial infarction [51]. Lui et al. think that hiPSC-derived CMs have been bioprinted to recapitulate a vascularized cardiac tissue which can then be transplanted in a defective heart [52]. Cho et al. used a 3D bioprinter to produce scaffold-free cardiac tissue grafts from hiPSC-derived CMs cell spheroids, and the results find that mechanical stretching stimulates hiPSC-derived CMs in a 3D printed, scaffold-free tissue graft to develop mature cardiac material structuring and cellular fates [53]. However, hiPSCs transplanted to lesions undergo oxidative damage and demonstrate squeeze loss as well as low cell survival and retention rates [54]. The development of biological 3D printing technology may overcome these problems to some extent. hiPSCs derived from the peripheral tissues of patients with disease specific mutations are a valuable tool to study the cardiac pathophysiology and drug development. The hiPSC-derived cardiac cells were successfully used to model cardiac diseases such as dilated cardiomyopathy and myocardial infarction [55]. The disease models help to identify the cellular phenotypes critical to cardiac pathology $[56,57]$.

Kupfer et al. used this bioink to 3D print hiPSC-laden structures with two chambers and a vessel inlet and outlet. After hiPSCs proliferated to a sufficient density, they differentiated the cells within the structure and demonstrated function of the resultant human chambered muscle pump. This advance represents a critical step toward generating macroscale tissues; human chambered organoids of this type might also serve as a testbed for cardiac medical devices and eventually lead to therapeutic tissue grafting [58].

When hiPSCs are separated into single cells, they are very sensitive to the process. Their pluripotency and directional differentiation ability are affected by many external factors, including printing methods, biological material, 


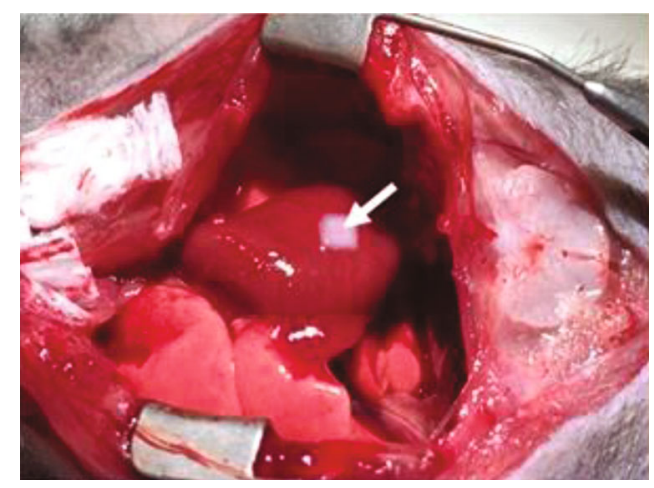

(a)

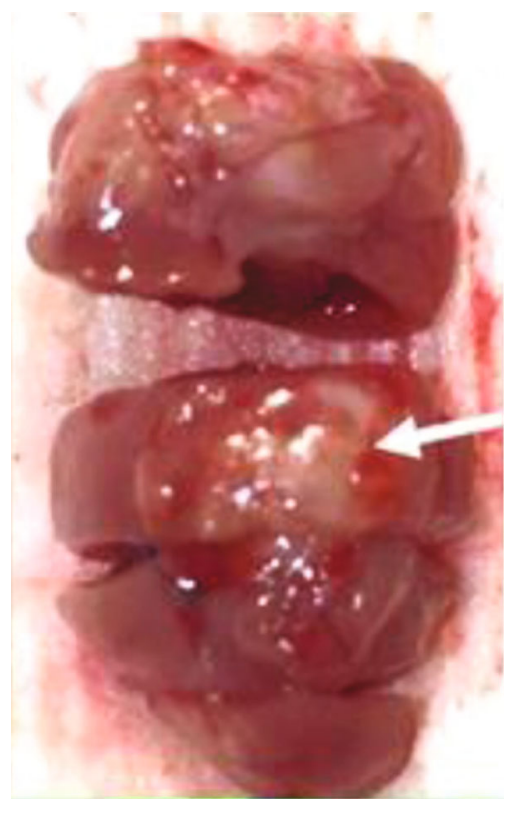

(c)

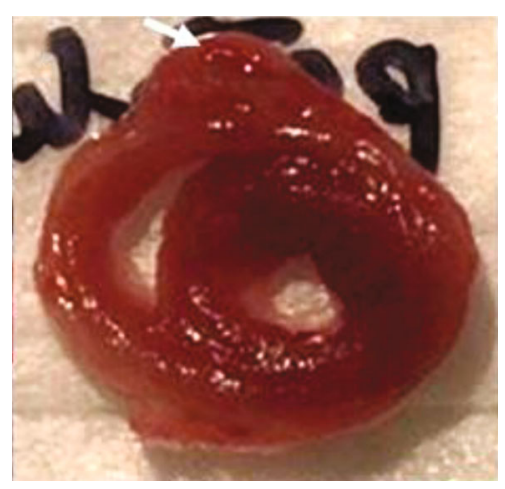

(b)

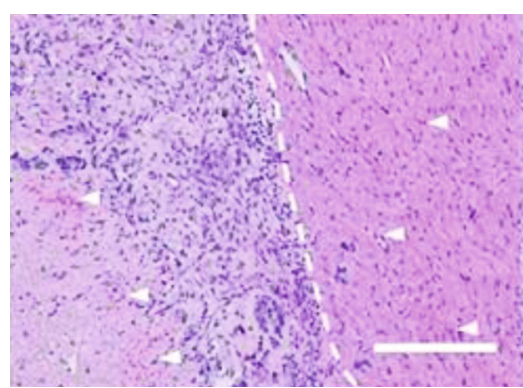

(d)

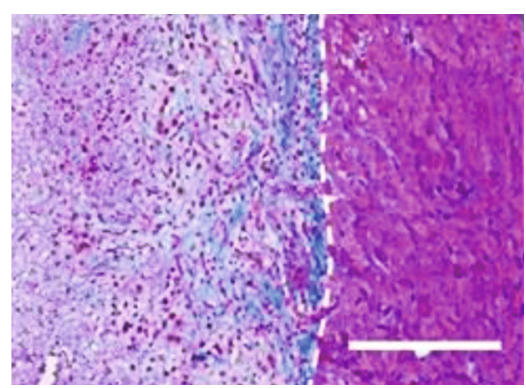

(e)

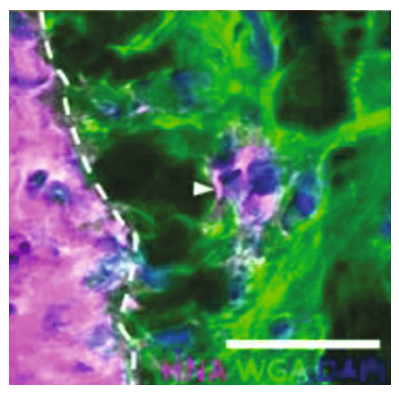

(f)

FIgURE 5: In vivo implantation of 3D bioprinted cardiac patches [13].

culture medium, and cell density [12]. A recent study used laser-assisted bioprinting technology to study the sensitivity of hiPSCs to biomaterials, such as collagen, alginate, hyaluronic acid, fibrinogen, fibrin, Geltrex ${ }^{\mathrm{TM}}$, Matrigel ${ }^{\mathrm{TM}}$, and cell culture medium. The results showed that bioinks with 85\% E8 and 15\% hyaluronic combined with Matrigel as a coating substrate provided optimal conditions for the survival and growth of hiPSCs [12]. Moreover, the study found that hiPSCs were not sensitive to laser printing itself, and cell survival was improved, and pluripotency was maintained after printing. In addition, directed differentiation showed that when hiPSCs were printed and induced to differentiate into CMs, they started to beat, demonstrating their functional cardiac phenotype. Some studies using hiPSCs derived from CMs to print directly [59]. Such studies described a method to produce photocrosslinkable tissue-specific decellularized extracellular matrix (dECM) bioinks for fabricating patient-specific tissues with high control over complex microarchitecture and mechanical properties achieved by a digital light processing-based scanningless and continuous 
TABLE 2: 3D bioprinting of iPSCs in tissue engineering applications.

\begin{tabular}{lccc}
\hline Application fields & Cell sources & Bioprinting outcomes & References \\
\hline Nerve tissue engineering & iPSCs & $\begin{array}{c}\text { Embryoid bodies containing endoderm, } \\
\text { ectoderm, and mesoderm cells }\end{array}$ & Gu et al. [12] \\
Bone tissue engineering & iPSCs & Cartilage & Nguyen et al. [45] \\
& iPSCs & CMs & Yoch et al. [23] \\
Cardiovascular tissue engineering & iPSCs & CMs/hepatocytes & Ong et al. [59] [13] \\
& hiPSCs, HUVECs, HCFs & Cardiac tissue sheets & Moldovan et al. [60] \\
& iPSCs & Vascular cells & Jones et al. [14] \\
iPSCs, hESCs & Hepatic markers & Ma et al. [9] & Hepatic markers \\
\hline
\end{tabular}

iPSCs: induced pluripotent stem cells; HCFs: human cardiac fibroblasts; hESCs: human embryonic stem cells; HUVECs: human umbilical vein endothelial cells.

3D bioprinter. They demonstrated that tissue-matched dECM bioinks provided a conducive environment for maintaining high viability and maturation of hiPSC-derived CMs and hepatocytes.

In addition, there were some interesting studies in the field of cardiovascular engineering that did not use bioinks. Recently, a protocol was established to obtain iPSC-derived endothelial progenitors (endothelial colony-forming cells) and smooth muscle-forming cells to assemble into vascular cell spheroids [60]. In these constructs, a layered distribution of alpha smooth muscle actin-positive cells and extracellular matrix deposition were achieved $[13,61]$. The same biological manufacturing method can also generate heart tissue. One study used hiPSC-derived vascular cell spheres as a novel cell material for scaffold-free biological construction. The spheres were composed of mixed human umbilical vein endothelial cells and hiPSC-derived CMs. Using this cell sheet technology, cardiac tissue sheets were generated, and the heart showed signs of vascularization when cardiac tissue sheets were implanted in rat hearts [13] (Figure 5). More research should be conducted to further study the construction of complex cardiac structures and functions of cardiovascular tissue by screening suitable biomaterials and improving the printing methods.

4.4. Applications for Liver Tissue Engineering. Liver transplantation is the only effective treatment for the end-stage liver disease. At present, the 5-year survival rate after liver transplantation has reached $73.6 \%$. However, the number of patients waiting for liver transplantation worldwide far exceeds the number of donors. The shortage of donor livers has become an important factor restricting the development of liver transplantation [62]. In-depth studies of liver regeneration and the developments in cell culture, stem cell technology, and genetic engineering should permit liver printing in the near future. One example is that researchers bioprinted hepatic tissue constructs using iPSC-derived hepatocytes, endothelial cells, and mesenchymal cells resuspended in two different bioinks: GelMA with stiffness similar to healthy liver tissues and a mix of glycidyl methacrylatehyaluronic acid/GelMA which supported vascularization $[63,64]$. However, liver printing has two crucial bottlenecks: the need to recreate a complicated internal system of branched blood vessels and the difficulty of distributing more than three high-density functional cells in a 3D structure and making them grow into tissues.

Chapin and Hajjar first attempted to produce iPSCderived liver bioprinting models. In their study, HLCs differentiated from both hiPSCs and hESCs were bioprinted and examined for the presence of hepatic markers to further validate the compatibility of the valve-based bioprinting process with the transfer of fragile cells [15]. The results showed that the examined cells were positive for nuclear factor 4 alpha, secreted albumin, and had morphology that was similar to that of hepatocytes. Both hESC and hiPSC lines were tested postprinting, and it was found that printed and nonprinted cells had negligible differences in terms of viability and pluripotency. To solve the bottlenecks of complex microstructures and different cell combinations in the liver microenvironment, one study used 3D bioprinting technology to construct a human liver model with a microstructure composed of hexagonal liver cell units and supporting cells that reflected structural and physiological features of the liver lobular array [9]. hiPSC-HPCs were embedded with human umbilical vein endothelial cells and adipose-derived stem cells in a microscale hexagonal architecture. This improved the functional properties and conformation of hiPSC-HPCs. Liver marker expression analysis showed that HPC maturity in the construct increased. To develop more physiological bioinks, this team mixed photocrosslinkable gelatin methacrylate and pig liver dECM. By adjusting the mechanical properties of the liver AECM bioink formula, the cell phenotype in the bioprinting construct could be improved, and the complex biochemical components of the liver phenotype were suitably maintained, which promoted the differentiation and maturation of hepatocytes derived from hiPSCs [59]. Liver organ printing technology has considerable potential and can provide patients with a specific platform for pathophysiology research, early drug screening, and clinical transformation.

4.5. Other Applications. In other study, a porous grid-type hiPSC-derived mesenchymal stem cell- (hi-MSC-) loaded hydrogel scaffold was constructed using a $3 \mathrm{D}$ bioprinting 
device. The 3D printed hydrogel scaffold provided a permissive in vitro living environment for hi-MSCs and significantly increased the survival duration of transplanted hiMSCs when compared with hi-MSCs administered locally in vivo. This study confirms that $3 \mathrm{D}$ printed hi-MSCloaded scaffold not only promoted the recovery of the endometrial histomorphology (endometrial tissue and gland regeneration) and the regeneration of endometrial cells (stromal cells and epithelial cells) and endothelial cells but also improved endometrial receptivity functional indicators [65]. In addition, hiPSCs that were generated from anterior cruciate ligament were used in the repair of ligaments and tendons [66].

\section{Conclusions}

Biological preparation of 3D printed stem cells is still a relatively novel research field, and various protocols for their manufacturing are in their infancy (Table 2). The clinical application of $3 \mathrm{D}$ printed stem cells has a long way to go and faces many challenges. The main reasons for the limited clinical application of these cells to date are related to the 3D printing methods, selection of biological materials, and acquisition of stem cells. The basic problems include printing speed, accuracy, survival rate, and cell viability. Bioinks as carriers should not only have optimal biocompatibility, degradability, and mechanical properties but also be suitable for mass production. The optimization of printing methods is another key aspect in achieving translation into the clinic.

The use of 3D printed iPSCs in the medical field is still in its preliminary stage. It has marked potential for use in tissue engineering and repair. Current research is mainly concentrated in the fields of orthopedic, neural, hepatic, and cardiovascular regeneration. Based on the continuous optimization of printing methods and biological inks, the use of multidirectional differentiation of the cells can achieve precise repair of complex structures. Simultaneously, the potential harm caused by multidirectional differentiation is also a point to be considered in future studies.

In summary, with the rapid development of stem cell 3D printing technology, biomaterials suitable for stem cell 3D printing are critical to its clinical application. Identification and validation of such materials will provide strong support for the realization of organ printing in the future and help solve the shortage of organ donors.

\section{Conflicts of Interest}

The authors declare that they have no conflict of interest.

\section{Authors' Contributions}

Dezhi Lu and Yang Liu are joint first authors.

\section{Acknowledgments}

The authors appreciate financial support from the National Key R\&D Program of China (2018YFA0703000/2018YFB1105600), the China Postdoctoral Science Foundation (Grant No.
2018M642042), the National Postdoctoral Program for Innovative Talents (Grant No. BX20180194), the National Natural Science Foundation of China (817723260/81902195), the Project of Shanghai Science and Technology Commission (18441903700/19XD1434200/18431903700), and the Twohundred Talent Support (20152224).

\section{References}

[1] S. Park, C. Lui, W. H. Jung et al., "Mechanical characterization of hiPSC-derived cardiac tissues for quality control," Advanced Biosystems, vol. 2, no. 12, 2018.

[2] G. Liu, et al.B. T. David, M. Trawczynski, and R. G. Fessler, "Advances in pluripotent stem cells: history, mechanisms, technologies, and applications," Stem Cell Reviews and Reports, vol. 16, no. 1, pp. 3-32, 2020.

[3] J. A. Reid, P. A. Mollica, G. D. Johnson, R. C. Ogle, R. D. Bruno, and P. C. Sachs, "Accessible bioprinting: adaptation of a low-cost 3D-printer for precise cell placement and stem cell differentiation," Biofabrication, vol. 8, no. 2, article 025017, 2016.

[4] M. Thomas and S. M. Willerth, "3-D bioprinting of neural tissue for applications in cell therapy and drug screening," Frontiers in Bioengineering and Biotechnology, vol. 5, 2017.

[5] K. Takahashi and S. Yamanaka, "Induction of pluripotent stem cells from mouse embryonic and adult fibroblast cultures by defined factors," Cell, vol. 126, no. 4, pp. 663-676, 2006.

[6] X. Wu, S. Mahalingam, S. K. VanOosten, C. Wisdom, C. Tamerler, and M. Edirisinghe, "New generation of tunable bioactive shape memory mats integrated with genetically engineered proteins," Macromolecular Bioscience, vol. 17, no. 2, 2017.

[7] F. Y. Hsieh, H. H. Lin, and S. H. Hsu, "3D bioprinting of neural stem cell-laden thermoresponsive biodegradable polyurethane hydrogel and potential in central nervous system repair," Biomaterials, vol. 71, pp. 48-57, 2015.

[8] L. Ho and S. H. Hsu, "Cell reprogramming by 3D bioprinting of human fibroblasts in polyurethane hydrogel for fabrication of neural-like constructs," Acta Biomaterialia, vol. 70, pp. 5770, 2018.

[9] J. C. Chapin, K. J. Hajjar, W. Zhu et al., "Fibrinolysis and the control of blood coagulation," Blood Reviews, vol. 29, no. 1, pp. 17-25, 2015.

[10] E. Abelseth, L. Abelseth, L. de la Vega, S. T. Beyer, S. J. Wadsworth, and S. M. Willerth, "3D printing of neural tissues derived from human induced pluripotent stem cells using a fibrin-based bioink," ACS Biomaterials Science and Engineering, vol. 5, no. 1, pp. 234-243, 2019.

[11] Y. Li, X. L. Jiang, L. Li et al., "3D printing human induced pluripotent stem cells with novel hydroxypropyl chitin bioink: scalable expansion and uniform aggregation," Biofabrication, vol. 10, no. 4, 2018.

[12] Q. Gu, E. Tomaskovic-Crook, G. G. Wallace, and J. M. Crook, "3D bioprinting human induced pluripotent stem cell constructs for in situ cell proliferation and successive multilineage differentiation," Advanced Healthcare Materials, vol. 6, no. 17, 2017.

[13] C. S. Ong, T. Fukunishi, H. T. Zhang et al., "Biomaterial-free three-dimensional bioprinting of cardiac tissue using human induced pluripotent stem cell derived cardiomyocytes," Scientific Reports, vol. 7, no. 1, 2017. 
[14] A. Faulkner-Jones, C. Fyfe, D. J. Cornelissen et al., "Bioprinting of human pluripotent stem cells and their directed differentiation into hepatocyte-like cells for the generation of mini-livers in 3D," Biofabrication, vol. 7, no. 4, 2015.

[15] J. C. Chapin and K. A. Hajjar, "Fibrinolysis and the control of blood coagulation," Blood Reviews, vol. 29, no. 1, pp. 17-24, 2015.

[16] L. de la Vega, D. A. R. Gómez, E. Abelseth, L. Abelseth, V. Allisson da Silva, and S. Willerth, "3D bioprinting human induced pluripotent stem cell-derived neural tissues using a novel lab-on-a-printer technology," Technology Applied Sciences, vol. 8, no. 12, p. 2414, 2018.

[17] R. Sharma, I. P. M. Smits, L. de la Vega, C. Lee, and S. M. Willerth, "3D bioprinting pluripotent stem cell derived neural tissues using a novel fibrin Bioink containing drug releasing microspheres," Frontiers in bioengineering and biotechnology, vol. $8,2020$.

[18] P. Kerscher, I. C. Turnbull, A. J. Hodge et al., "Direct hydrogel encapsulation of pluripotent stem cells enables ontomimetic differentiation and growth of engineered human heart tissues," Biomaterials, vol. 83, pp. 383-395, 2016.

[19] M. Robinson, S. Douglas, and S. Michelle Willerth, "Mechanically stable fibrin scaffolds promote viability and induce neurite outgrowth in neural aggregates derived from human induced pluripotent stem cells," Scientific Reports, vol. 7, no. 1, p. 6250, 2017.

[20] X. Ma, X. Qu, W. Zhu et al., "Deterministically patterned biomimetic human iPSC-derived hepatic model via rapid 3D bioprinting," Proceedings of the National Academy of Sciences, vol. 113, no. 8, pp. 2206-2211, 2016.

[21] H. J. Kim, K. K. Kim, I. K. Park, B. S. Choi, J. H. Kim, and M. S. Kim, "Hybrid scaffolds composed of hyaluronic acid and collagen for cartilage regeneration," Tissue Engineering and Regenerative Medicine, vol. 9, no. 2, pp. 57-62, 2012.

[22] R. Noguchi, K. Nakayama, M. Itoh et al., "Development of a three-dimensional pre-vascularized scaffold-free contractile cardiac patch for treating heart disease," The Journal of Heart and Lung Transplantation, vol. 35, no. 1, pp. 137-145, 2016.

[23] L. Koch, A. Deiwick, A. Franke et al., "Laser bioprinting of human induced pluripotent stem cells-the effect of printing and biomaterials on cell survival, pluripotency, and differentiation," Biofabrication, vol. 10, no. 3, 2018.

[24] J. Kapr, L. Petersilie, T. Distler et al., "Human induced pluripotent stem cell-derived neural progenitor cells produce distinct neural 3D in vitro models depending on alginate/gellan gum/laminin hydrogel blend properties," Advanced Healthcare Materials, vol. 10, no. 16, 2021.

[25] M. Raasch, E. Fritsche, A. Kurtz, M. Bauer, and A. S. Mosig, "Microphysiological systems meet hiPSC technology - new tools for disease modeling of liver infections in basic research and drug development," Advanced Drug Delivery Reviews, vol. 140, pp. 51-67, 2019.

[26] R. Noguchi, K. Nakayama, M. Itoh et al., "Development of a three-dimensional pre-vascularized scaffold-free contractile cardiac patch for treating heart disease," Journal of Heart and Lung Transplantation, vol. 35, no. 1, pp. 137-145, 2016.

[27] L. Gao, M. E. Kupfer, J. P. Jung et al., "Myocardial tissue engineering with cells derived from human-induced pluripotent stem cells and a native-like, high-resolution, 3-dimensionally printed scaffold," Circulation Research, vol. 120, no. 8, pp. 1318-1325, 2017.
[28] S. J. Song, Y. C. Shin, S. E. Kim et al., "Aligned laminin corepolydioxanone/collagen shell fiber matrices effective for neuritogenesis," Scientific Reports, vol. 8, no. 1, 2018.

[29] S. W. Carlson, H. Yan, M. Ma, Y. Li, J. Henchir, and C. E. Dixon, "Traumatic brain injury impairs soluble $\mathrm{N}$ ethylmaleimide-sensitive factor attachment protein receptor complex formation and alters synaptic vesicle distribution in the hippocampus," Journal of Neurotrauma, vol. 33, no. 1, pp. 113-121, 2016.

[30] K. Li, Y. Jiang, and D. Jiang, "Experimental study of vein subvolution combined with neural stem cells to repair sciatic neurologic defects in rats," International Journal of Clinical and Experimental Pathology, vol. 8, no. 9, pp. 10941-10946, 2015.

[31] K. Song, W. Li, and M. Li, “Acute promyelocytic leukemia following autologous bone marrow-derived mesenchymal stem cell transplantation for traumatic brain injury: a case report," Oncology Letters, vol. 10, no. 5, pp. 2905-2908, 2015.

[32] B. De Strooper and E. Karran, "The cellular phase of Alzheimer's disease," Cell, vol. 164, no. 4, pp. 603-615, 2016.

[33] S. Vijayavenkataraman, S. Thaharah, S. Zhang, W. F. Lu, and J. Y. H. Fuh, "3D-Printed PCL/rGO Conductive scaffolds for peripheral nerve injury repair," Artificial Organs, vol. 43, no. 5, pp. 515-523, 2019.

[34] S. M. Chambers, C. A. Fasano, E. P. Papapetrou, M. Tomishima, M. Sadelain, and L. Studer, "Highly efficient neural conversion of human ES and iPS cells by dual inhibition of SMAD signaling," Nature Biotechnology, vol. 27, no. 3, pp. 275-280, 2009.

[35] F. Zhou, L. Zhang, L. Chen et al., "Prevascularized mesenchymal stem cell-sheets increase survival of random skin flaps in a nude mouse model," American Journal of Translational Research, vol. 11, no. 3, pp. 1403-1416, 2019.

[36] M. Hirano, Y. Huang, D. Vela Jarquin et al., “3D bioprinted human iPSC-derived somatosensory constructs with functional and highly purified sensory neuron networks," Biofabrication, vol. 13, no. 3, 2021.

[37] R. Lin, C. Deng, X. Li et al., "Copper-incorporated bioactive glass-ceramics inducing anti-inflammatory phenotype and regeneration of cartilage/bone interface," Theranostics, vol. 9, no. 21, pp. 6300-6313, 2019.

[38] M. Varela-Eirin, J. Loureiro, E. Fonseca et al., "Cartilage regeneration and ageing: targeting cellular plasticity in osteoarthritis," Ageing Research Review, vol. 42, pp. 56-71, 2018.

[39] H. Kwon, W. E. Brown, C. A. Lee et al., "Surgical and tissue engineering strategies for articular cartilage and meniscus repair," Nature Reviews Rheumatology, vol. 15, no. 9, pp. 550-570, 2019.

[40] M. Tu, Y. Yao, F. H. Qiao, and L. Wang, "The pathogenic role of connective tissue growth factor in osteoarthritis," Bioscience Reports, vol. 39, no. 7, 2019.

[41] R. Chijimatsu, M. Kobayashi, K. Ebina et al., "Impact of dexamethasone concentration on cartilage tissue formation from human synovial derived stem cells in vitro," Cytotechnology, vol. 70, no. 2, pp. 819-829, 2018.

[42] T. S. Lynch, R. M. Patel, A. Benedick, N. H. Amin, M. H. Jones, and A. Miniaci, "Systematic review of autogenous osteochondral transplant outcomes," Arthroscopy, vol. 31, no. 4, pp. 746-754, 2015.

[43] S. Duchi, C. Onofrillo, C. D. O'Connell et al., "Handheld CoAxial Bioprinting: Application to in situ surgical cartilage repair," Scientific Reports, vol. 7, no. 1, 2017. 
[44] C. Meinert, K. Schrobback, D. W. Hutmacher, and T. J. Klein, "A novel bioreactor system for biaxial mechanical loading enhances the properties of tissue-engineered human cartilage," Scientific Reports, vol. 7, no. 1, 2017.

[45] D. Nguyen, D. A. Hägg, A. Forsman et al., "Cartilage Tissue Engineering by the 3D Bioprinting of iPS Cells in a Nanocellulose/Alginate Bioink," Scientific Reports, vol. 7, no. 1, p. 658, 2017.

[46] R. Gulati, A. Behfar, J. Narula et al., "Acute myocardial infarction in young individuals," Mayo Clinic Proceedings, vol. 95, no. 1, pp. 136-156, 2020.

[47] G. Scrascia, C. Rotunno, P. Guida et al., "Haemostasis alterations in coronary artery bypass grafting: comparison between the off-pump technique and a closed coated cardiopulmonary bypass system," Interactive cardiovascular and thoracic surgery, vol. 16, no. 5, pp. 636-642, 2013.

[48] M. Mirramezani, S. L. Diamond, H. I. Litt, and S. C. Shadden, "Reduced order models for transstenotic pressure drop in the coronary arteries," Journal of biomechanical engineering, vol. 141, 2019.

[49] W. Yan, E. Abu-el-Rub, S. Saravanan, L. A. Kirshenbaum, R. C. Arora, and S. Dhingra, "Inflammation in myocardial injury: mesenchymal stem cells as potential immunomodulators," American Journal of Physiology Heart and Circulatory Physiology, vol. 317, no. 2, pp. H213-H225, 2019.

[50] K. L. Wang, Q. Xue, X. H. Xu, F. Hu, and H. Shao, "Recent progress in induced pluripotent stem cell-derived 3D cultures for cardiac regeneration," Cell and Tissue Research, vol. 384, no. 2, pp. 231-240, 2021.

[51] B. Yang, C. Lui, E. Yeung et al., "A net mold-based method of biomaterial-free three-dimensional cardiac tissue creation," Tissue Engineering Part C: Methods, vol. 25, no. 4, pp. 243252, 2019.

[52] C. Lui, A. F. Chin, S. Park et al., "Mechanical stimulation enhances development of scaffold-free, 3D-printed, engineered heart tissue grafts," Journal of Tissue Engineering and Regenerative Medicine, vol. 15, no. 5, pp. 503-512, 2021.

[53] S. Cho, C. Lee, M. A. Skylar-Scott, S. C. Heilshorn, and J. C. $\mathrm{Wu}$, "Reconstructing the heart using iPSCs: engineering strategies and applications," Journal of Molecular and Cellular Cardiology, vol. 157, pp. 56-65, 2021.

[54] Y. J. Choi, H. G. Yi, S. W. Kim, and D. W. Cho, “3D cell printed tissue analogues: a new platform for theranostics," Theranostics, vol. 7, no. 12, pp. 3118-3137, 2017.

[55] Y. Yoshida and S. Yamanaka, "Induced pluripotent stem cells 10 years later: for cardiac applications," Circulation Research, vol. 120, no. 12, pp. 1958-1968, 2017.

[56] J. T. Hinson, A. Chopra, N. Nafissi et al., "Titin mutations in iPS cells define sarcomere insufficiency as a cause of dilated cardiomyopathy," Science, vol. 349, no. 6251, pp. 982-986, 2015.

[57] M. Tiburcy, J. E. Hudson, P. Balfanz et al., "Defined engineered human myocardium with advanced maturation for applications in heart failure modeling and repair," Circulation, vol. 135, no. 19, pp. 1832-1847, 2017.

[58] M. E. Kupfer, W. H. Lin, V. Ravikumar et al., "In situ expansion, differentiation, and electromechanical coupling of human cardiac muscle in a 3D bioprinted, chambered organoid," Circulation Research, vol. 127, no. 2, pp. 207-224, 2020.
[59] C. Yu, X. Y. Ma, W. Zhu et al., "Scanningless and continuous 3D bioprinting of human tissues with decellularized extracellular matrix," Biomaterials, vol. 194, pp. 1-13, 2019.

[60] L. Moldovan, A. Barnard, C. H. Gil et al., "iPSC-derived vascular cell spheroids as building blocks for scaffold-free biofabrication," Biotechnology Journal, vol. 12, no. 12, 2017.

[61] C. S. Ong, T. Fukunishi, A. Nashed et al., "Creation of cardiac tissue exhibiting mechanical integration of spheroids using 3D bioprinting," Jove-Journal of Visualized Experiments, vol. 125, 2017.

[62] J. Chow, M. Hantes, J. Houle, and C. Zalavras, "Arthroscopic autogenous osteochondral transplantation for treating knee cartilage defects: a 2- to 5-year follow-up study," Arthroscopy, vol. 20, no. 7, pp. 681-690, 2004.

[63] S. Cho, C. Lee, M. A. Skylar-Scott, S. C. Heilshorn, and J. C. $\mathrm{Wu}$, "Reconstructing the heart using iPSCs: Engineering strategies and applications," Journal of Molecular and Cellular Cardiology, vol. 157, pp. 56-65, 2021.

[64] T. Billiet, M. Vandenhaute, J. Schelfhout, S. van Vlierberghe, and P. Dubruel, "A review of trends and limitations in hydrogel-rapid prototyping for tissue engineering," Biomaterials, vol. 33, no. 26, pp. 6020-6041, 2012.

[65] W. Ji, B. Hou, W. Lin et al., "3D bioprinting a human iPSCderived MSC-loaded scaffold for repair of the uterine endometrium," Acta Biomaterialia, vol. 116, pp. 268-284, 2020.

[66] N. Ashammakhi, A. GhavamiNejad, R. Tutar et al., "Highlights on advancing frontiers in tissue engineering," Tissue Engineering Part B: Reviews, 2021. 